\title{
Nonlinear vibration of rotating system near resonance
}

\author{
G. Sypniewska-Kamińska ${ }^{1}$, R. Starosta ${ }^{1}$, and J. Awrejcewicz ${ }^{2}$ \\ ${ }^{1}$ Poznan University of Technology, Institute of Applied Mechanics, Piotrowo 3, 60-965 Poznań, Poland \\ ${ }^{2}$ Technical University of Lodz, Department of Automatics and Biomechanics Sciences, Stefanowskiego 90-924, Łódź, \\ Poland
}

\begin{abstract}
The paper concerns analysis of nonlinear vibration of the rotating systems consisted of rigid disks mounted on the elastic massless shaft. The investigations are focused on their behaviour under the resonance conditions. The analytical method of multiple scales in time domain is applied to the analysis of dynamics of the system near main resonance. The transition phenomenon depending on the value of the nonlinearity parameter is discussed.
\end{abstract}

\section{Introduction}

Torsional vibration belongs to major problems in design of the power transmission systems. The dynamic stresses caused by torsional oscillations, especially near resonance when the amplitudes grow significantly, may be very large and can lead to failure of the whole system.

Both discrete and continuous models are commonly used in order to investigate the torsional vibration of the power transmission systems $[1,4,6]$.

The problem of vibration in nonlinear systems has to be treated with due care. The numerical methods commonly used in solving nonlinear problems do not create the possibility of a synthetic regard on the diversity of the dynamic behaviour of the systems. Some surprising types of the vibrational motion may be not detected when only the numerical methods are used. The Multiple Scales Method (MSM) in time domain, belonging to the asymptotic methods, not only allows obtain the approximate solutions, but also gives the possibility to exact investigate the influence of the particular parameters on the motion. This method is applied in the paper to solve the problem of torsional vibration of the shaft on which the rigid disks are mounted. The relationships, obtained in the analytical form, between the amplitudes and phases of vibration at resonance have been examined. Associating the MSM with an interesting approach proposed by Manevich [3] known as Limiting Phase Trajectory (LPT) Method allows to predict the sudden change of the shape of the amplitude modulation which appears in resonance at a certain value of the nonlinearity parameter. A similar approach, but based completely on the proposed by Manevich formulation in the field of complex functions, was applied to investigate the torsional vibration in the system with one degree of freedom [5].

\section{The description of the system}

Two rotating and vibrating systems presented in figure 1 are considered in the paper. The systems consist of two or three disks fasten to the elastic shaft. The shaft is relatively light, so its mass may be neglected. Elastic properties of the shaft are nonlinear of the cubic type. Therefore, two coefficients of stiffness, marked by $K_{j}$ and $K_{j n}$, are introduced for the $j$-th part of the shaft. Moreover, viscous damping is taken into account, and $C_{j}$ for $j=1,2$ denotes the damping coefficient. The whole system is mounted on frictionless bearings which are also ideal in the geometric sense. One of the disks is under the action of the harmonically changing torque $M(t)=M_{0} \cos (P t)$.

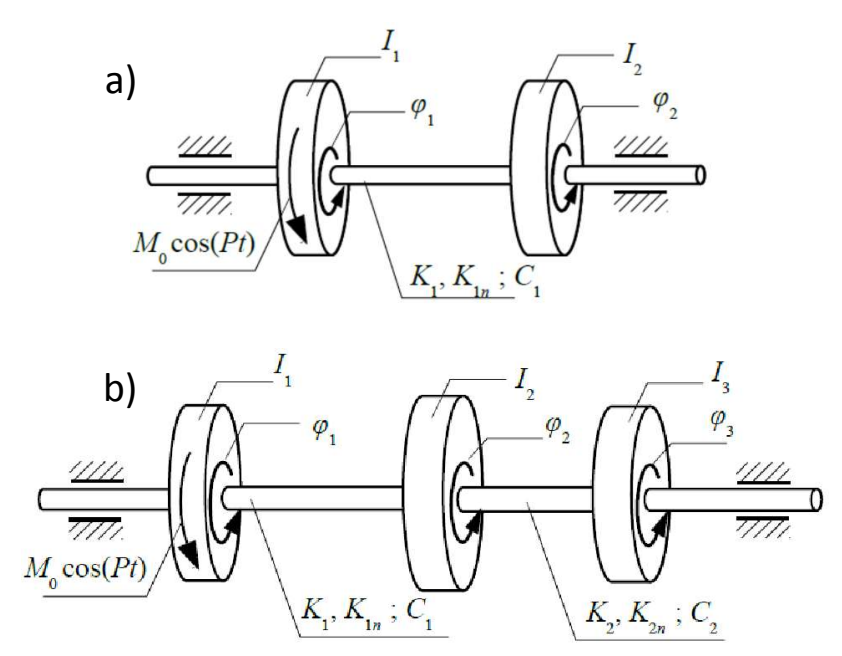

Fig. 1. Model of rotating systems with a) two and b) three disks. 


\section{Mathematical model}

The systems have two or three degrees of freedom, respectively. The rotation angles $\varphi_{j}$ of the $j$-th wheels are chosen as the generalized coordinates. The Lagrange formalism is used to derive the mathematical model describing the system.

The Lagrangian of the system in the most general form is

$L=\frac{1}{2} \sum_{j=1}^{N} I_{j} \dot{\varphi}_{j}^{2}-\frac{1}{2} \sum_{j=1}^{N-1} K_{j}\left(\varphi_{j}-\varphi_{j+1}\right)^{2}$

$-\frac{1}{4} \sum_{j=1}^{N-1} K_{j n}\left(\varphi_{j}-\varphi_{j+1}\right)^{4}$

where $I_{j}$ is the moment of inertia of the $j$-th disk and $N$ is the total number of them.

The external excitation and damping effects are included into mathematical model as the generalized forces. The equations of motion derived from the Lagrange equation of the second type are transformed into dimensionless form.

In order to investigate the vibration, it is very convenient to introduce the function describing the internal motion i.e. the screw angle of the $j$-th shaft

$\Phi_{j}(t)=\varphi_{j}(t)-\varphi_{j+1}(t), \quad j=1, \ldots, N-1$.

In this way we exclude from calculation the rigid rotation of the shaft and further investigation concerns only the vibrational effects.

The governing equation for the system with two disks takes the form of the Duffing equation

$\ddot{\phi}_{1}(\tau)+c_{1} \dot{\phi}_{1}(\tau)+\phi_{1}(\tau)+\alpha_{1} \phi_{1}(\tau)^{3}=m_{0} \cos p \tau$.

For the case with three disks the governing equations are

$\ddot{\phi}_{1}+c_{1} \dot{\phi}_{1}+\phi_{1}+\alpha_{1} \phi_{1}^{3}-c_{2} \gamma_{3} \dot{\phi}_{2}-\gamma_{3} w^{2} \phi_{2}-\alpha_{2} \gamma_{3} \phi_{2}^{3}$

$=m_{0} \cos (p \tau)$,

$\ddot{\phi}_{2}+c_{2} \dot{\phi}_{2}+w^{2} \phi_{2}+\alpha_{2} \phi_{2}^{3}-c_{1} \gamma_{1} \dot{\phi}_{1}-\gamma_{1} \phi_{1}-\alpha_{1} \gamma_{1} \phi_{1}^{3}=0$,

where $\quad m_{0}=M_{0} /\left(I_{1} k_{1}\right), \quad p=P / \sqrt{k_{1}}, \quad c_{j}=\hat{c}_{j} / \sqrt{k_{1}}$, $\gamma_{3}=I_{3} /\left(I_{3}+I_{2}\right), \quad \gamma_{1}=I_{1} /\left(I_{1}+I_{2}\right), \quad \alpha_{j}=k_{j n} / k_{1}$, $w=k_{2} / k_{1}$, dimensionless time $\tau=\sqrt{k_{1}} t, \quad$ and $k_{j}=K_{j}\left(I_{j}+I_{j+1}\right) /\left(I_{j} I_{j+1}\right), k_{j n}=K_{j n}\left(I_{j}+I_{j+1}\right) /\left(I_{j} I_{j+1}\right)$, $\hat{c}_{j}=C_{j}\left(I_{j}+I_{j+1}\right) /\left(I_{j} I_{j+1}\right)$ for $j=1, \ldots, N-1$.

The equations (3) and (4) - (5) are supplemented by the initial conditions

$\phi_{j}(0)=\phi_{0 j}, \dot{\phi}_{j}(0)=\beta_{0 j}, \quad j=1, \ldots, N-1$

where $\phi_{0 j}$ and $\beta_{0 j}$ are known values.

\section{Asymptotic solution}

The initial value problems are solved in the asymptotic way, using Multiple Scales Method (MSM) in time domain. Let us assume that the system is weakly nonlinear and the damping coefficient and the amplitude of the external torque are also of order of the small parameter $\varepsilon$ $m_{0} \rightarrow \widetilde{m}_{0} \varepsilon, \alpha_{j} \rightarrow \widetilde{\alpha}_{j} \varepsilon, c_{j} \rightarrow \widetilde{c}_{j} \varepsilon$.

The assumed form of the asymptotic solution is as follows

$\phi_{j}(\tau)=\phi_{j 0}\left(\tau_{0}, \tau_{1}\right)+\varepsilon \phi_{j 1}\left(\tau_{0}, \tau_{1}\right)$,

where $\tau_{0}$ and $\tau_{1}$ are slow and fast time scales, respectively.

The differential operators relating to these time scales take the form

$\frac{d}{d \tau}=\frac{\partial}{\partial \tau_{0}}+\varepsilon \frac{\partial}{\partial \tau_{1}}$,

$\frac{d^{2}}{d \tau^{2}}=\frac{\partial^{2}}{\partial \tau_{0}^{2}}+2 \varepsilon \frac{\partial^{2}}{\partial \tau_{0} \partial \tau_{1}}+o\left(\varepsilon^{2}\right)$

Then relations (7) - (9) are introduced into equations of motion (3) or (4) - (5). In this way, the small parameter appears in the model. After arranging the equation with respect to the power of the small parameter, the equations of the first and the second order approximation are obtained.

\subsection{Case I - shaft with two disks}

The analysis is concerned on the case of the main resonance, that occurs when $p \approx 1$. In order to consider this case, the small detuning parameter $\sigma$ is introduced in the form $p=1+\sigma=1+\varepsilon \tilde{\sigma}$.

After substituting (7) - (9) into Eq. (3) and arranging it with respect to the powers of the small parameter $\varepsilon$ we obtain

- the equation of order $\varepsilon^{0}$

$\frac{\partial^{2} \phi_{10}}{\partial \tau_{0}^{2}}+\phi_{01}=0$

- the equation of order $\varepsilon^{1}$

$\frac{\partial^{2} \phi_{11}}{\partial \tau_{0}^{2}}+\phi_{11}=\widetilde{m}_{0} \cos \left(1+\widetilde{\sigma} \tau_{1}\right)-$

$\widetilde{\alpha}_{1} \phi_{10}^{3}-\widetilde{c}_{1} \frac{\partial \phi_{10}}{\partial \tau_{0}}-2 \frac{\partial^{2} \phi_{10}}{\partial \tau_{0} \partial \tau_{1}}$.

The solution of the first order equation (10) is introduced into Eq. (11).

We are interested in the bounded solutions, so all secular terms ought to vanish. Zeroing the secular terms leads to the solvability condition

$\frac{d A\left(\tau_{1}\right)}{d \tau_{1}}+3 \widetilde{\alpha}_{1} A^{2}\left(\tau_{1}\right) \bar{A}\left(\tau_{1}\right)+i \widetilde{c}_{1} A\left(\tau_{1}\right)=\frac{1}{2} \widetilde{m}_{0} e^{i \tau_{1} \tilde{\sigma}}$,

where $A\left(\tau_{1}\right)$ is the unknown complex function.

It is convenient to express the function $A\left(\tau_{1}\right)$ by the real-valued functions $a\left(\tau_{1}\right), \psi\left(\tau_{1}\right)$ describing amplitude and phase respectively

$A\left(\tau_{1}\right)=\frac{1}{2} a\left(\tau_{1}\right) e^{i \psi\left(\tau_{1}\right)}$.

Taking advantage of relations (9) and (13), and returning to the original denotations of the system parameters, one can obtain 
$\frac{d a}{d \tau}+i a \frac{d \psi}{d \tau}+\frac{1}{2} c_{1} a-\frac{3}{8} i \alpha_{1} a^{3}=\frac{m_{0}}{2} e^{i(\sigma \tau)} e^{-i \psi}$.

Separating in (14) real and imaginary parts, we can write $\frac{d a}{d \tau}+\frac{1}{2} c_{1} a=\frac{m_{0}}{2} \cos \theta$

$-a \frac{d \theta}{d \tau}+a \sigma-\frac{3}{8} \alpha_{1} a^{3}=\frac{m_{0}}{2} \sin \theta$,

where $\theta=\sigma \tau-\psi$ is the modified phase. Equations (15) describe the modulation of the amplitude $a$ and modified phase $\theta$.

Let us consider the non-damped vibrations $\left(c_{1}=0\right)$. In that case, the set of Eqs (15) has the first integral

$H=-a \frac{m_{0}}{2} \sin \theta+\sigma \frac{a^{2}}{2}-\frac{3}{32} \alpha_{1} a^{4}=$ const,

where the constant $H$ depends on the initial conditions. Equation (16) represents one-parameter family of the curves on the plane $(a, \theta)$. We are especially interested in the case when maximal energy exchange between the system and the external loading appears. This situation takes place for $H=0$. In that case the first integral (16) has the form:

$-16 m_{0} \sin \theta+16 \sigma a-3 \alpha_{1} a^{3}=0$.

It is easily to show that the curve given by Eq. (17) has extrema for $\theta=-\pi / 2$ and $\theta=\pi / 2$. The qualitative change in the behaviour of the system is observable for the critical value of the nonlinearity parameter

$\alpha_{c}=\frac{64 \sigma^{3}}{81 m_{0}^{2}}$.

The trajectories on the plane $(a, \theta)$ for various values of $\alpha_{1}$ are presented and discussed in details in paper [5].

When $\alpha_{1}$ exceeds the critical value $\alpha_{c}$, the shape of the amplitude modulation rapidly changes. The amplitude modulation in time $\tau$, obtained from Eqs (15), is presented in figures 2 and 3 for $\alpha_{1}$ a bit smaller, and a bit greater than $\alpha_{c}$.

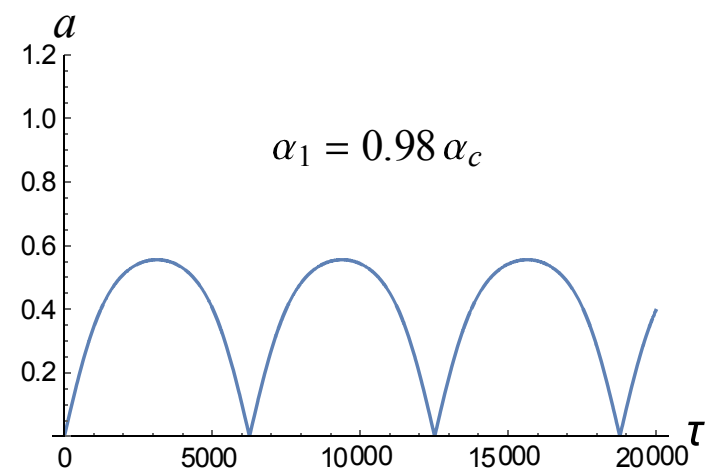

Fig. 2. Amplitude modulation for $\alpha_{1}$ just below $\alpha_{c} \quad(\sigma=0.01$, $\left.m_{0}=0.002, \phi_{01}=0, \beta_{01}=0\right)$.

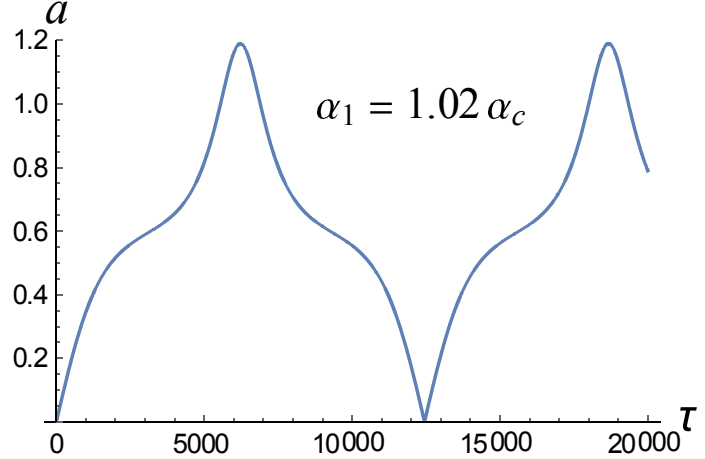

Fig. 3. Amplitude modulation for $\alpha_{1}$ just above $\alpha_{c} \quad(\sigma=0.01$,

$$
\left.m_{0}=0.002, \phi_{01}=0, \beta_{01}=0\right) \text {. }
$$

The transition which occurs at $\alpha_{c}$ indicates stepwise change in the intensity of energy exchange between the system and external loading.

\subsection{Case II - shaft with three discs}

The procedure applied to solve the problem is in general similar but more complicated to that one in the case I. Now the equations of order $\varepsilon^{0}$ reads

$$
\begin{aligned}
& \frac{\partial^{2} \phi_{10}}{\partial \tau_{0}^{2}}+\phi_{10}-\gamma_{3} w^{2} \phi_{20}=0 \\
& \frac{\partial^{2} \phi_{20}}{\partial \tau_{0}^{2}}+w^{2} \phi_{20}-\gamma_{1} \phi_{10}=0
\end{aligned}
$$

and the equations of order $\varepsilon^{1}$ are

$$
\begin{aligned}
& \frac{\partial^{2} \phi_{11}}{\partial \tau_{0}^{2}}+\phi_{11}-\gamma_{3} w^{2} \phi_{21}=-\widetilde{\alpha}_{1} \phi_{10}{ }^{3}+\tilde{\alpha}_{2} \gamma_{3} \phi_{20}{ }^{3}-\widetilde{c}_{1} \frac{\partial \phi_{10}}{\partial \tau_{0}}+ \\
& \widetilde{c}_{2} \gamma_{3} \frac{\partial \phi_{20}}{\partial \tau_{0}}-2 \frac{\partial^{2} \phi_{10}}{\partial \tau_{0} \partial \tau_{1}}+\tilde{m}_{0} \cos \left(p \tau_{0}\right),
\end{aligned}
$$

$$
\begin{aligned}
& \frac{\partial^{2} \phi_{21}}{\partial \tau_{0}^{2}}+w^{2} \phi_{21}\left(\tau_{0}, \tau_{1}\right)-\gamma_{1} \phi_{11}\left(\tau_{0}, \tau_{1}\right)=\tilde{\alpha}_{1} \gamma_{1} \phi_{10}\left(\tau_{0}, \tau_{1}\right)^{3}- \\
& \tilde{\alpha}_{2} \phi_{20}\left(\tau_{0}, \tau_{1}\right)^{3}+\widetilde{c}_{1} \gamma_{1} \frac{\partial \phi_{10}}{\partial \tau_{0}}-\widetilde{c}_{2} \frac{\partial \phi_{20}}{\partial \tau_{0}}-2 \frac{\partial^{2} \phi_{20}}{\partial \tau_{0} \partial \tau_{1}} .
\end{aligned}
$$

The linear differential operator in Eqs (19) - (20) has two different self-frequencies

$$
\begin{aligned}
& \omega_{1}=\sqrt{\frac{1+w^{2}-\sqrt{1-2 w^{2}+w^{4}+4 w^{2} \gamma_{1} \gamma_{3}}}{2}}, \\
& \omega_{2}=\sqrt{\frac{1+w^{2}+\sqrt{1-2 w^{2}+w^{4}+4 w^{2} \gamma_{1} \gamma_{3}}}{2}} .
\end{aligned}
$$

The linear combination of the normal modes forms the solution of the first order equations. It can be written in the exponential form

$$
\begin{aligned}
& \phi_{10}=A_{11}\left(\tau_{1}\right) e^{i \tau_{0} \omega_{1}}+A_{12}\left(\tau_{1}\right) e^{i \tau_{0} \omega_{2}}+C C, \\
& \phi_{20}=\frac{1-\omega_{1}^{2}}{\gamma_{3} w^{2}} A_{11}\left(\tau_{1}\right) e^{i \tau_{0} \omega_{1}}+\frac{1-\omega_{2}^{2}}{\gamma_{3} w^{2}} A_{12}\left(\tau_{1}\right) e^{i \tau_{0} \omega_{2}}+C C,
\end{aligned}
$$


where $A_{1 j}\left(\tau_{1}\right)$ for $j=1,2$ are unknown complex functions and $C C$ denotes the complex conjugate parts.

It is convenient to introduce the real-valued functions $a_{1 j}\left(\tau_{1}\right), \psi_{1 j}\left(\tau_{1}\right), j=1,2$, similarly as in the previous section (see Eq. (13)).

Let us consider the case $p \approx \omega_{1}$ of main resonance. The detuning parameter $\sigma$ is introduced in the form $p=\omega_{1}+\sigma=\omega_{1}+\varepsilon \tilde{\sigma}$.

The requirement of zeroing the secular terms in the equations (20) leads to the modulation equation

$$
\begin{aligned}
& \frac{d}{d \tau} a_{1 j}(\tau)=F_{j}\left(\alpha_{1}, \alpha_{2}, c_{1}, c_{2}, w, \gamma_{1}, \gamma_{3}, m_{0}, \sigma_{1}\right), j=1,2 \\
& \frac{d}{d \tau} \psi_{1 j}(\tau)=G_{j}\left(\alpha_{1}, \alpha_{2}, c_{1}, c_{2}, w, \gamma_{1}, \gamma_{3}, m_{0}, \sigma_{1}\right), j=1,2
\end{aligned}
$$

where functions $F$ and $G$ can be expressed explicitly.

The amplitude modulation obtained from Eqs (25) (26) are presented in figures 4 and 5.

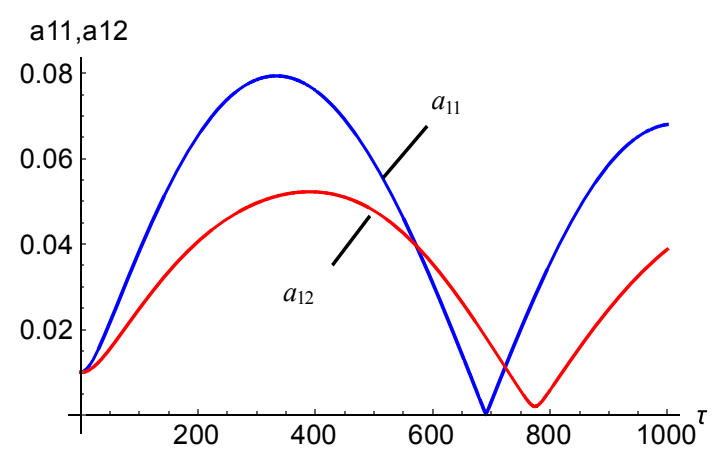

Fig. 4. The modulation of amplitude for $\phi_{10}(\tau)$ related to the first and second natural frequency $\left(\alpha_{1}=0.4, \alpha_{2}=0.15\right.$, $c_{1}=c_{2}=0.001 \gamma_{1}=0.57, \gamma_{3}=2.5, \sigma=0.01, m_{0}=0.001$, $\left.\phi_{01}=0, \beta_{01}=0\right)$.

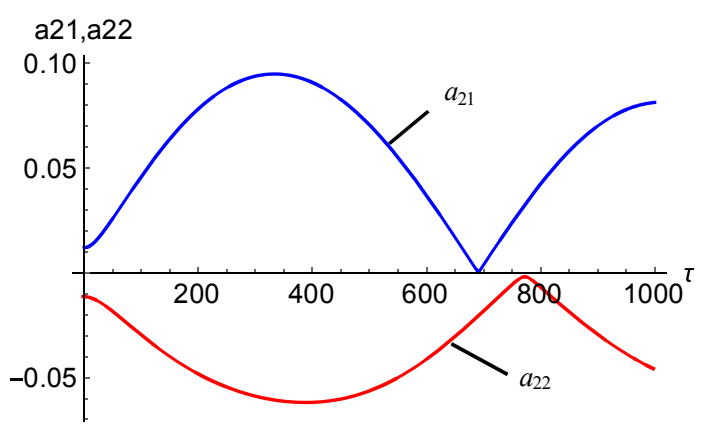

Fig. 5. The modulation of amplitude for $\phi_{20}(\tau)$ related to the first and second natural frequency $\left(\alpha_{1}=0.4, \alpha_{2}=0.15\right.$, $c_{1}=c_{2}=0.001 \gamma_{1}=0.57, \gamma_{3}=2.5, \sigma=0.01, m_{0}=0.001$,

$$
\left.\phi_{01}=0, \beta_{01}=0\right) \text {. }
$$

The first mode vibration is connected with frequency $\omega_{1}$ given in Eq. (21) and the second with frequency $\omega_{2}$ given in Eq. (22).

\section{Conclusions}

Analysis of the nonlinear disks-shaft system has been done using the asymptotical Multiple Scales Method in time domain properly adopted to solve the problem.

The investigation has been focused mainly on the non-steady vibration of the forced system near the external resonance.

The main advantage of the asymptotic solutions consists in achieving qualitative information on the dynamics of the considered systems. The important nonlinear dynamical transition has been detected for the critical value of the parameter connected with the spring nonlinearity in the case with two disks. The amplitudes modulation indicate the intensive energy exchange between the system and external excitation.

The second of considered problems which concerns the system reducible to the mathematical model of two degree of freedom is significantly more complicated and requires further studies. This model is strongly coupled what requires to introduce some modification into the classical formulation of the MSM. The eigenvalues of the differential operator i.e. the undamped natural frequencies are determined in the paper. The modulation equations for the amplitude and phase of the normal modes in case of the main resonance are also obtained.

\section{Acknowledgements}

This paper was financially supported by the National Science Centre of Poland under the grant MAESTRO 2, No. 2012/04/A/ST8/00738, for years 2013-2016.

\section{References}

1. Y.Huang Ying, S.Yang, F.Zhang, C.Zhao, Q.Ling, and H.Wang, Chinese J. Mech. Eng. 25, 4 (2012)

2. J. Kožešnik, Dynamics of machines (in Polish), (WNT, 1963)

3. L. I. Manevitch, A.I. Musienko, Nonlinear Dyn. 58 (2009)

4. B. Porter, J. Mech. Eng. Sci. 7, 1 (1965)

5. R. Starosta, Nonlinear dynamics of discrete systems - asymptotic approach (in Polish), $\mathrm{Nr} 457$ Rozprawy, WPP, Poznań 2011

6. Z.Wenming, W.Bohua, Z.Shuangshuang and L.Shuang, Int. J. Com. Sci. 10, 3 (2013) 